\title{
Damage formation on fused silica illuminated with ultraviolet-infrared femtosecond pulse pairs
}

\author{
Xiaoming Yu ${ }^{\mathrm{a}}$, Zenghu Chang ${ }^{\mathrm{b}}$, Paul B. Corkum ${ }^{\mathrm{c}, \mathrm{d}}$, and Shuting Lei ${ }^{\mathrm{a}^{*}}$ \\ ${ }^{a}$ Industrial and Manufacturing Systems Engineering, Kansas State University, Manhattan, \\ Kansas 66506, USA \\ ${ }^{\mathrm{b}}$ CREOL and Department of Physics, University of Central Florida, Orlando, Florida 32816, \\ USA \\ ${ }^{\mathrm{c}}$ National Research Council of Canada, Ottawa, Ontario K1A 0R6, Canada \\ ${ }^{\mathrm{d}}$ University of Ottawa, Ottawa, Ontario K1N 6N5, Canada \\ *lei@ksu.edu; phone 1785 532-3731
}

\begin{abstract}
We investigate damage formation on the surface of fused silica by two femtosecond laser pulses, a tightly focused $266 \mathrm{~nm}$ (UV) pulse followed by a loosely focused $800 \mathrm{~nm}$ (IR) pulse. We show that the damage size is determined by the UV pulse, and only a small fraction of the normal UV damage threshold energy is needed to cause damage when combined with the properly delayed IR pulse. Our results, analyzed with a rate equation model, suggest that the UV pulse generates seed electrons through multiphoton absorption and the IR pulse utilizes these electrons to cause damage by avalanche ionization. By tuning such parameters like pulse energy, time delay, IR pulse duration and polarization, we further demonstrate that damage profile can be controlled.
\end{abstract}

Keywords: femtosecond laser machining, UV pulse, IR pulse, multiphoton ionization, avalanche ionization, fused silica

\section{INTRODUCTION}

Optical control of electronic excitation is at the core of ultrafast laser modification of wide-bandgap materials [1], and holds the key to future technologies such as petahertz (PHz) signal processing [2]. Due to their high intensities $\left(>10^{14} \mathrm{~W} / \mathrm{cm}^{2}\right)$, focused femtosecond laser beams can promote electrons from the valance band to the conduction band, through ionization processes such as multiphoton, tunneling and impact (avalanche) ionizations [3, 4]. These ionization processes can lead to a rapid increase of conduction-band (free) electrons, the density of which determines whether modification and damage can be eventually generated [5]. Single femtosecond laser beams have been used extensively in studying the interaction between femtosecond laser and solid materials, and both new phenomena and novel applications continue to be discovered.

However, as far as practical applications are concerned, the use of single beams may not offer the full potential of ultrafast lasers, and is therefore not ideal in terms of energy efficiency and overall effectiveness. First, concerning energy efficiency, short-wavelength (such as ultraviolet and extreme-ultraviolet) pulses are needed in machining applications where nanoscale focal spots are required $[6,7]$. Such short-wavelength pulses are usually generated by harmonic generation procedures, which suffer from low conversion efficiency. For example, in our third-harmonicgeneration setup, we convert fundamental, $4 \mathrm{~mJ}, 800-\mathrm{nm}$ pulses into 266-nm pulses with the maximum pulse energy of $20 \mu \mathrm{J}$, resulting in a conversion efficiency of $0.5 \%$ [8]. Generating extreme-ultraviolet (XUV) pulses by high harmonic generation (HHG) has an even lower efficiency [9]. It is, therefore, desired to design a machining technique with which low-cost long-wavelength pulses can be used to assist the high-cost short-wavelength pulses, provided that the advantages of using short-wavelengths (e.g., reduced focal spot size) can be retained.

Second, even if conversion efficiency could be greatly improved with future technologies, and UV pulse with high pulse energies could be obtained at low costs, combination with another pulse at a different wavelength is still 
required in order to improve overall effectiveness. For example, compared with IR pulses, UV pulses are known to have higher multiphoton ionization rates (because of their higher photon energy), but lower avalanche ionization rates [10]. While multiphoton ionization is indispensable in the buildup of initial free electrons in wide-band-gap materials, avalanche ionization is much more effective in producing more free electrons due to its single-photonabsorption nature. Therefore, combining UV with IR pulses can utilize both of their advantages and dramatically increase the free electron density.

In this paper, we report experimental and simulation results of femtosecond laser machining with UV-IR pulse pairs. While the use of multiple pulses in the ablation of materials has been reported [11], and the effect of increased ablation rate and improved morphologies have been shown [12-14], we focus our research specifically on the reduction of UV damage threshold with the assistance of IR pulses without sacrificing UV beam's spatial resolution. In Section 2, we first investigate the relationship between UV damage thresholds and IR pulse energies at various UV-IR delays. In Section 3 we tightly focus the UV beam and demonstrate that nano-scale feature can be achieved with significantly reduced UV pulse energy. Our results suggest that further improvement in machining resolution can be achieved with short-wavelength (such as XUV) pulses with pulse energies well below their normal damage threshold. The control of electron dynamics demonstrated in this paper provides another dimension of tuning femtosecond laser ablation of dielectrics, and may lead to new fundamental discoveries and novel applications.

\section{TWO-COLOR MACHINING ON THE MICROMETER SCALE}

\subsection{Reduction of UV damage threshold}

In the first series of experiments, we investigate the reduction of UV pulse damage threshold with the assistance of the IR pulse [8]. We choose to focus both UV and IR beams with low-NA lenses in order to check damage shape and size conveniently with optical microscopes. The experimental setup is shown in Fig. 1. Our femtosecond laser system delivers $60 \mathrm{fs}$ (full width at half maximum, FWHM) laser pulses at the center wavelength of $800 \mathrm{~nm}$ with the maximum pulse energy of $4.3 \mathrm{~mJ}$ and repetition rate of $1 \mathrm{kHz}$. The beam is split into two arms (UV and IR) after a beam splitter. $90 \%$ of the beam is reflected (UV arm) and directed through a third harmonic generation (THG) module (Eksma Optics), which generates 266-nm wavelength pulses with an estimated pulse duration of 70 fs and pulse energy of $20 \mu \mathrm{J}$. The UV pulse energy is adjusted by a neutral-density (ND) filter before the first BBO. After the THG module, only the UV beam is efficiently reflected by two dichroic mirrors (DM1 and DM2, Layertec) mounted on a motor-driven delay stage. Then the UV beam is focused by a lens (50 mm focal length). The $800 \mathrm{~nm}$ beam in the IR arm is focused by another lens ( $200 \mathrm{~mm}$ focal length), and its energy is controlled by an ND filter.

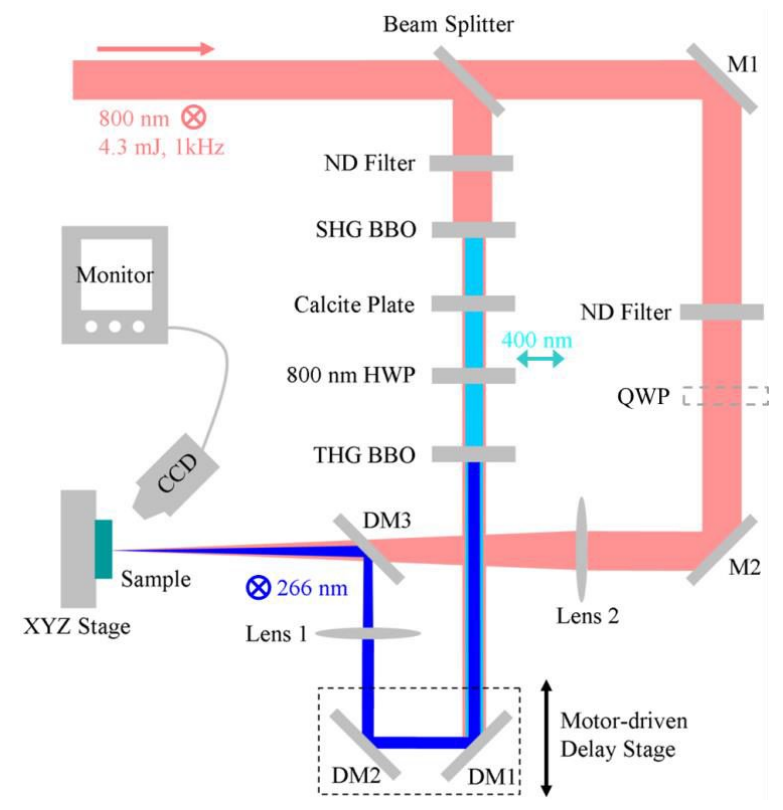

Fig. 1. Experimental setup. 
The focused UV and IR beams are combined with another dichroic mirror (DM3) and both foci are carefully aligned on the sample surface. The samples are square fused silica plates of $25.4 \mathrm{~mm}$ wide and $2 \mathrm{~mm}$ thick (Corning 7980). The samples are mounted on a motor-driven three-axis stage. To temporally overlap the UV and IR beams, a 200$\mu \mathrm{m}$ thick BBO crystal is inserted after DM3 and the difference frequency generation of $400 \mathrm{~nm}$ from the UV and IR is observed. Zero delay is defined when the generated $400 \mathrm{~nm}$ light is the strongest, with an accuracy of $\pm 30 \mathrm{fs}$. In this experiment, both UV and IR beams have linear polarizations (pointing into the paper plane, marked in Fig. 1). For the experiments reported in Section 2.2, an 800-nm quarter waveplate (QWP) is inserted in the IR arm to obtain either linear or circular polarizations for the IR pulse.

In the experiments, both the UV and IR energy fluence are kept below their respective damage threshold $\left(0.87 \mathrm{~J} / \mathrm{cm}^{2}\right.$ for UV and $2.17 \mathrm{~J} / \mathrm{cm}^{2}$ for IR), so that no visible damage is observed with a charge-coupled device (CCD) camera when only one beam is applied. At a fixed IR fluence and a relative delay to the UV pulse, UV energy is carefully adjusted (still kept below its damage threshold) to a level that visible damages are starting to appear, and this UV energy is defined as the UV damage threshold energy.

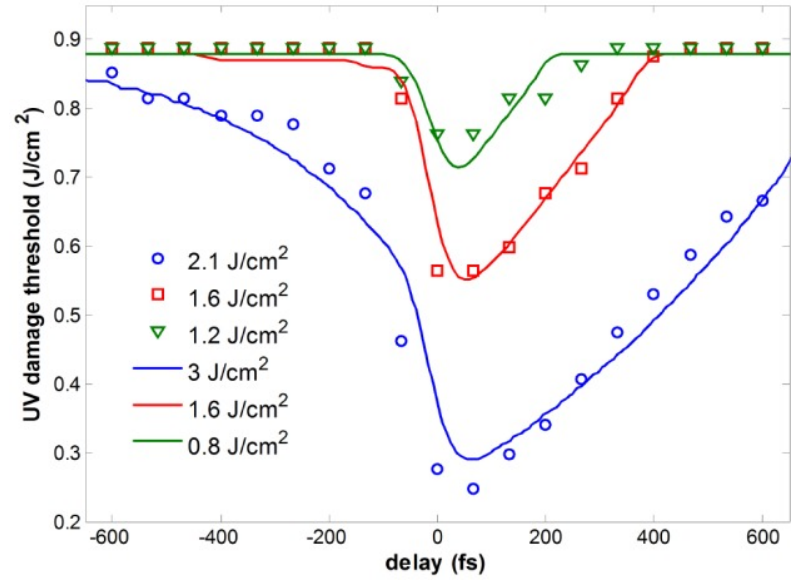

Fig. 2. Dependence of UV damage threshold on the time delay at different IR fluences. Dots: experimental data. Curves: simulation data.

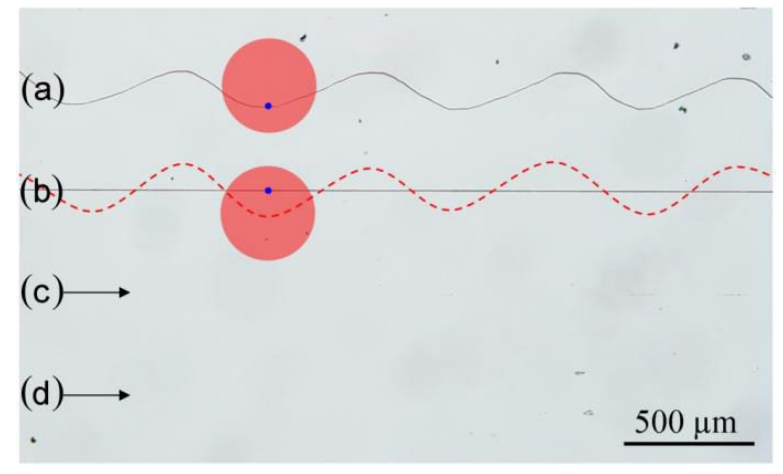

Fig. 3. Optical images of different structures fabricated by a combination of UV and IR pulses. (a) Moving UV spot while keeping IR spot fixed. (b) Moving IR spot while keeping UV spot fixed. No structure can be seen using either (c) UV or (d) IR beam individually to

Figure 2 shows the dependence of the UV damage threshold on time delay between the UV and IR pulses for different IR fluences. Positive values in delay means UV pulses are irradiated on sample surfaces before IR pulses. At the highest IR fluence, the UV damage threshold reaches a minimum of $0.25 \mathrm{~J} / \mathrm{cm}^{2}$ at $60 \mathrm{fs}$ delay, which is $71 \%$ lower than that in the normal case (damage threshold by UV alone). Actually, it is found that if IR fluence is kept at $2 \%$ below its damage threshold, the lowest fluence needed at zero delay for UV is $88 \%$ lower than that in the normal case. Away from this "optimal" delay, UV damage threshold increases, however, in an asymmetric manner: It returns to the normal value faster from the negative delay side than from the positive delay side. With IR fluence decreasing, more UV energy is required to damage the sample, i.e., the "effective" damage threshold increases.

In Fig. 2, for every IR fluence, the UV threshold reaches a minimum value at $\sim 60 \mathrm{fs}$ and increases faster in the negative delay direction than in the positive delay direction. Since the number of free electron density reaches the maximum at the end of the $60 \mathrm{fs}$ UV pulse because the lifetime of the electrons is much longer than the laser pulse duration, the minimum UV fluence is found at the $60 \mathrm{fs}$ delay. Because UV is more efficient in generating seed electrons, the asymmetric shape is seen at each IR fluence. It is noted that the UV damage threshold is higher at zero delay. This is probably because the free electrons need some time to build up, and the maximum number is reached after the UV pulse. Another feature seen from this figure is the dependence of UV threshold on IR fluence. A higher IR fluence provides more avalanche ionization and can re-excite more free electrons from STEs, and therefore, a lower UV threshold is observed. It is found that with a high IR fluence $\left(2.1 \mathrm{~J} / \mathrm{cm}^{2}\right)$, the UV damage threshold returns to its normal value at a delay of $\sim 27.3 \mathrm{ps}$, which is similar to the lifetime of STEs in accordance with previous results [15]. 
Since damage forms as a combined effect of UV and IR pulses, the damage size is determined by the size of the area where the two beam overlap. In Fig. 3(a), the IR spot size is deliberately enlarged by moving its focus forward by 8.2 $\mathrm{mm}$, resulting in a $330 \mu \mathrm{m}$ diameter spot on the fused silica surface. The UV is focused on the surface and in the central portion of the IR spot. The sample moves towards left at a constant speed of $0.5 \mathrm{~mm} / \mathrm{s}$, and while moving, DM3 is tilted vertically back and forth so that a wavy structure is written on the sample surface. On the contrary, in Fig. 3(b), while keeping the UV spot fixed and tilting M2, the position of the IR spot is changed vertically and a straight line is written. The temporal delay difference by tilting those mirrors is calculated to be less than $1 \mathrm{fs}$. Since the seeding beam such as the UV or XUV can be focused to a spot much smaller than that of IR, this paves the way for machining features on scales that cannot be achieved with IR beam alone.

\subsection{The role of IR polarizations}

Next we investigate the effect of IR polarization on the UV damage threshold. The experimental setup used in this experiment is similar to that shown in Fig. 1, except that an $800-\mathrm{nm}$ zero-order quarter waveplate is inserted in the IR arm to change it to either linear or circular polarization. The UV pulse remains linearly polarized throughout this experiment. We first apply UV and IR beams individually and check their damage shapes, as shown in Fig. 4. For the IR pulse, circularly shaped damages are generated, and their sizes decrease with decreasing pulse energy. Comparing these two IR polarizations, we observe a slightly larger damage size for the linear polarization. Plotting the squared radii against the logarithm of pulse energies (Fig. 5) reveals nearly linear relationship for both IR polarizations.

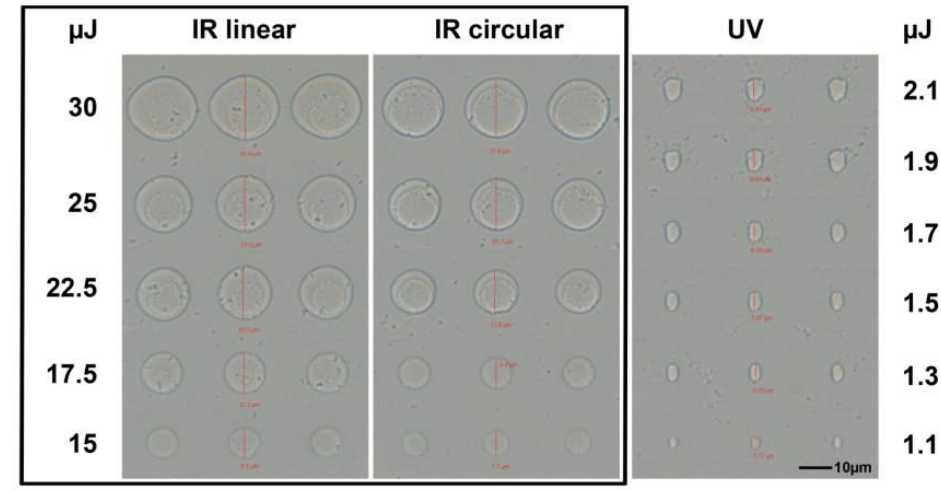

Fig. 4. Optical images of single-shot damages generated by IR (with linear and circular polarizations) and UV (with linear polarization) pulses when applied individually. Three damage spots are shown for each condition. Pulse energies for IR and UV are shown on the left and right side, respectively.

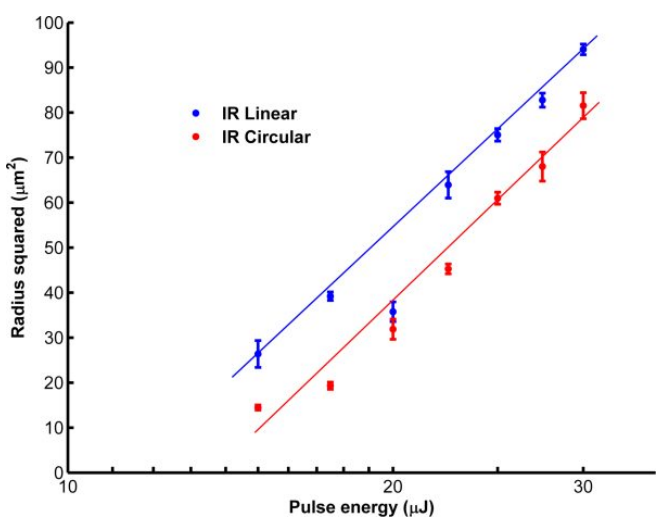

Fig. 5. Relationship between squared radius $\left(r^{2}\right)$ and pulse energy $(E)$ for the IR pulse. All of the lines are guide to the eye.

In the literature, the damage threshold energy is often determined as the energy value at which these straight lines intersect with the $\mathrm{x}$-axis [16]. However, as has been pointed out [17], this method only applies to single-photon absorption, whereas in our experiment the initial buildup of free electrons via IR and UV's multiphoton ionization is a 6and 2-photon process, respectively. Nevertheless, the consistently smaller damage size for the circular polarization at each energy level suggests a higher damage threshold compared to the linear polarization. Our intention throughout this paper is to use the IR pulse to assist the UV pulse, without damaging the sample by the IR pulse itself. Therefore, the higher damage threshold for the circular polarization provides us the opportunity to increase the IR pulse energy, and this might further reduce the UV damage threshold (results shown below).

The damages generated by the UV pulse show an oval shape, as shown in Fig. 4, perhaps due to the difference of diverging angles between the vertical and horizontal directions, and this problem can be solved with spatial shaping techniques (demonstrated in Section 3.2). While not ideal for machining purposes, such oval shape can be beneficial in the current study, because after the combined action of UV and IR pulses, resultant damages with similar oval shape would suggest that the damage is indeed "seeded" by the UV pulse. The $r^{2} \sim \log (E)$ plot for the UV pulse is shown in Fig. 6 . 
To compare the two IR polarization states, we combine UV and IR pulses at zero delay and use various UV and IR pulse energies. We use 15 different UV energies ranging from 0.05 to $2.8 \mu \mathrm{J}$, and $5 \mathrm{IR}$ conditions, i.e., $10 \mu \mathrm{J}$ linear and circular polarizations, $12.5 \mu \mathrm{J}$ linear and circular polarizations, and $15 \mu \mathrm{J}$ circular polarization. No damage is observed with only the IR beam at each condition. The IR condition $15 \mu \mathrm{J}$ linear polarization is excluded from this result because during this experiment damage is observed with the IR beam itself at this condition. This differs from the results reported in Fig. 4, where damage is observed at $15 \mu \mathrm{J}$ with both polarizations, perhaps due to a slight misplacement of the sample surface outside of the IR focal plane, resulting in a slightly increased IR spot size on the sample surface and thus a lower fluence. We measure the damage diameter along the vertical (long-axis) direction, and the $r^{2} \sim \log (E)$ relationship is shown in Fig. 6, with corresponding optical images shown on the left.
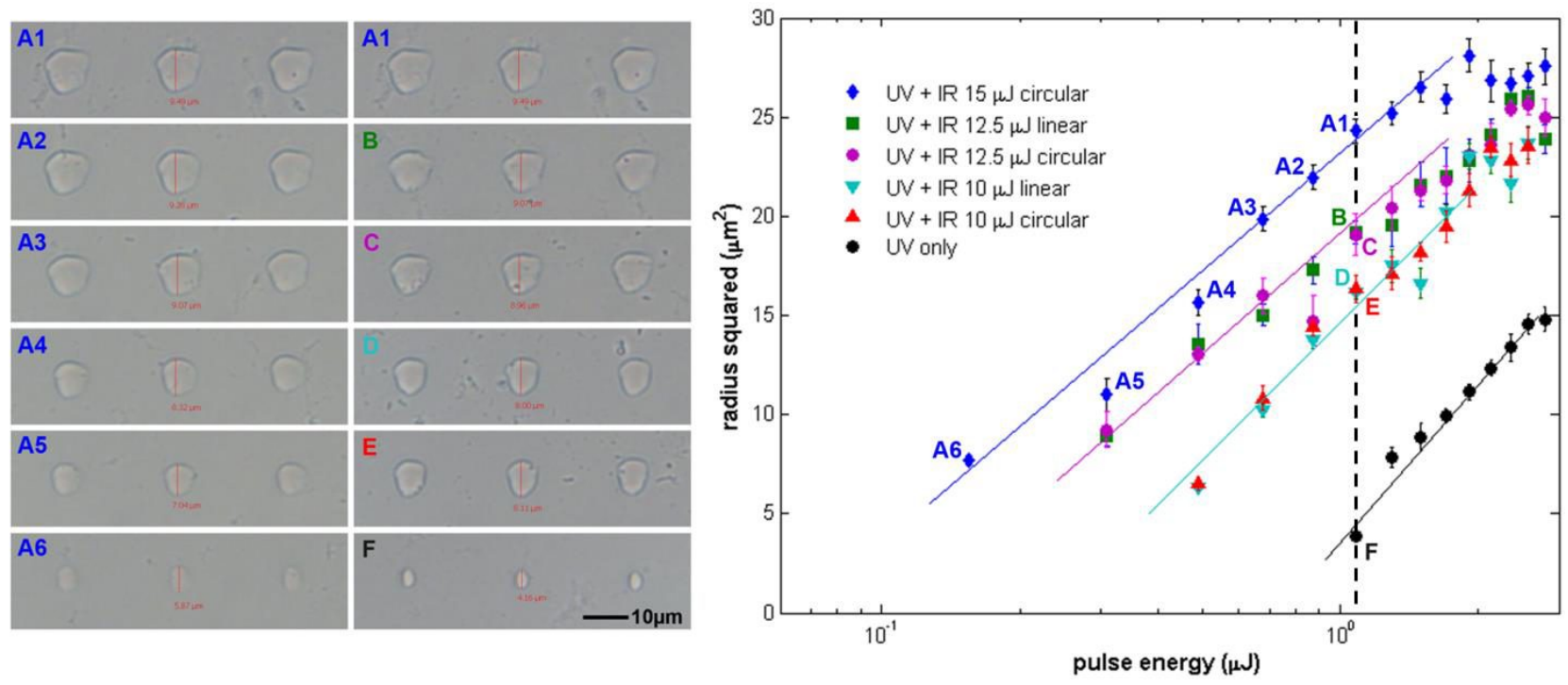

Fig. 6. Relationship between squared radius (r2) and UV pulse energy (E), and optical images for select data points. Points A1, B, C, D, E and F are at the same UV pulse energy. All of the lines are guide to the eye.

First, comparing the UV only with all the other cases with a certain UV energy at and above $1.1 \mu \mathrm{J}$ (the A1-F line), we clearly observe the increase of damage size, indicating the reduction of UV damage threshold. In fact, no UV only damage is seen below $1.1 \mu \mathrm{J}$ (the A1-F line), and all the data points on the left side of the A1-F line indicate damage formation with the help of the IR pulse. Second, with the same IR condition, e.g., $15 \mu \mathrm{J}$ circular polarization (A1-A6), damages show oval shape (especially at low UV energies), indicating that it is the UV pulse that provides the seeding electrons. The relationship between $r^{2}$ and $\log (E)$ follows a straight line with a slope similar to the UV only case. Third, since our purpose is to investigate the effect of two IR polarizations, we pay special attention to the four IR conditions $(10$ and $12.5 \mu \mathrm{J})$. Between these conditions (e.g., points B and C, and points D and E), we observe no apparent difference in either damage shape or size. This is perhaps due to the fact that, despite different multiphoton ionization rates for linear and circular polarizations, with the same pulse energy (and therefore the same intensity), electric fields in linearly and circularly polarized beams accelerate free electrons to the same kinetic energy, which leads to an equal probability for impact ionization [18], and thus both polarizations have the same avalanche ionization rate. And finally, due to the higher damage threshold for the circularly polarized IR beam, a higher IR pulse energy can be supplied to the UV pulse, and the UV threshold can be further reduced, as seen from the "UV+IR $15 \mu \mathrm{J}$ circular" data. As mentioned above, the $r^{2} \sim \log (E)$ plot may give erroneous damage threshold for wide bandgap materials, and therefore is not used for this study. Instead, we define an energy range within which damage threshold will fall. For example, for the two 12.5 $\mu \mathrm{J}$ cases, damage is seen at $0.31 \mu \mathrm{J}$, but not at the next lower energy level $0.16 \mu \mathrm{J}$. Therefore, the damage threshold at this condition must lie between 0.16 to $0.31 \mu \mathrm{J}$. Similarly, the threshold for the $15 \mu \mathrm{J}$ case lies between 0.05 and $0.16 \mu \mathrm{J}$. As a result, further reduction of UV damage threshold is achieved with circularly polarized IR pulses.

\section{TWO-COLOR MACHINING ON THE NANOMETER SCALE}

Besides the reduction of UV damage threshold, another goal is to demonstrate that the damage size is controlled by the seeding short-wavelength UV pulse. This is important in machining applications where small feature is required. 


\subsection{Approaching nano-scale}

The experimental setup in this section is similar to that shown in Fig. 1, except that the lens used to focus the UV beam is replaced by a reflecting objective with a numerical aperture (NA) of 0.5 , and the IR beam is directed to the sample surface with an angle of $60^{\circ}$ to the surface normal. The details of the setup can be found elsewhere [19].
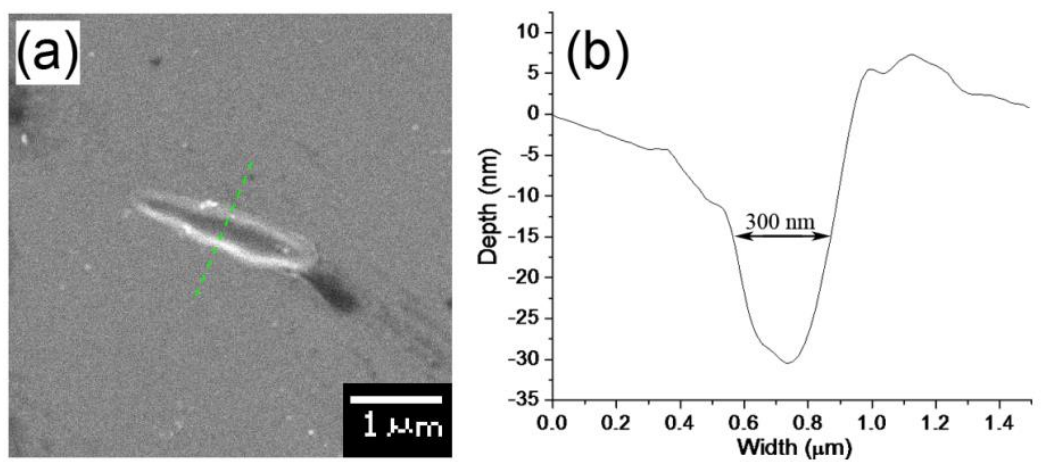

Fig. 7. (a) SEM image of a damage spot caused by single UV beam. (b) Cross section along the dashed line in (a). The pulse energy is $64 \mathrm{~nJ}$.

First we examine the damage caused by a single UV beam. Figure 7 shows a damage spot caused by the single UV beam with pulse energy of $64 \mathrm{~nJ}$. The damage is a line shape instead of a round one, which is attributed to the following two factors. First, the beam quality is not good enough, especially the outer portion of the beam that the reflecting objective lens uses to focus. This has been experimentally verified with a separate experiment (results not shown here). Second, the beam profile of the THG beam is not round. Right after the THG BBO, the beam size $\left(1 / \mathrm{e}^{2}\right)$ is $2.9 \mathrm{~mm}$ (horizontal) and $3.9 \mathrm{~mm}$ (vertical) measured by the knife-edge method assuming a Gaussian profile. Due to the nonlinearity of the interaction between the laser pulse and the sample, the resulting damage becomes more elliptical. To obtain a $300 \mathrm{~nm}$ circular damage spot, we suggest to use a UV transmitting objective to fully utilize the central portion of the beam. In addition, a pair of cylindrical lenses (a concave and a convex lens) can be inserted after the THG BBO to correct the asymmetric profile, and spatial filtering can also be used to improve the beam quality. The cross section along the narrow direction of Fig. 7(a) is shown in Fig. 7(b). The FWHM width is measured to be $300 \mathrm{~nm}$.

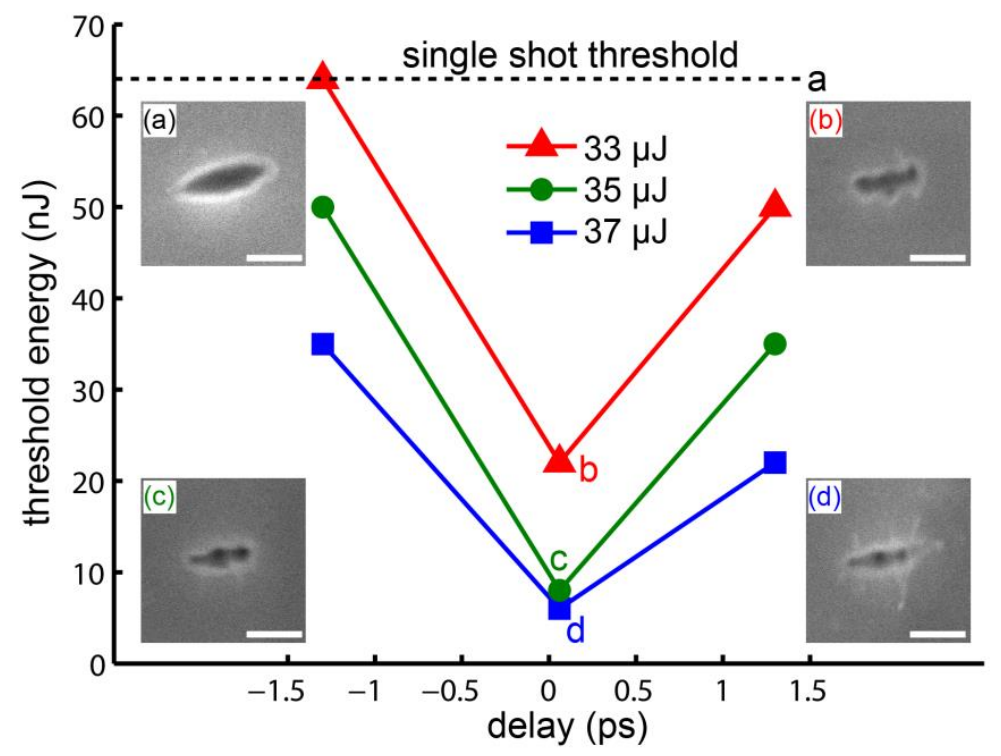

Fig. 8. Relationship between threshold energy and delay of UV and IR pulses. Insets: SEM images of each point indicated by a, b, c and d. Scale bars are $1 \mu \mathrm{m}$. Black dashed line indicates UV single beam threshold. The solid lines are guides to the eye. 
With the combination of UV and IR beams, the damage threshold of UV beam can be lowered by $~ 90 \%$, as shown in Fig. 8. Three IR energy levels ( $33 \mu \mathrm{J}, 35 \mu \mathrm{J}, 37 \mu \mathrm{J})$ and three delays (-1.3 ps, $60 \mathrm{fs}, 1.3 \mathrm{ps})$ are used. Positive delays are defined as when the UV pulse reaches the sample before the IR pulse. In the experiments, different UV energy is used and the damage threshold is determined as the lowest energy with which nanoscale damage is observed using SEM. No damage is seen using only the IR beam. As can be seen in Fig. 8, at 60 fs delay and $37 \mu \mathrm{J} \mathrm{IR} \mathrm{energy,} \mathrm{UV} \mathrm{damage}$ threshold is $\sim 10 \%$ of the normal value, and it increases with longer delays and with lower IR energy. In contrast to the multiple shot threshold, this time we used single shot threshold measurement to avoid incubation effects. It should be noted that at $60 \mathrm{fs}$ delay, the damage threshold using IR energy of $37 \mu \mathrm{J}$ and $35 \mu \mathrm{J}$ is similar. This is because $6 \mathrm{~nJ}$ is the lowest energy level that can be measured accurately due to background noise. Therefore, the actual damage threshold using IR energy of $37 \mu \mathrm{J}$ may be lower than $6 \mathrm{~nJ}$. The insets show SEM images of the points indicated by the corresponding letters. As can be seen, the feature size is similar for different cases, which suggests that this technique can be used for nanomachining with an XUV (or shorter wavelength) beam combined with a longer wavelength beam, and the resulting feature size is only determined by the XUV beam. It should be noted that with the combination of two beams, there is an increase of edge roughness in morphology (Figure 8(b)-(d)) compared to the damage caused by single UV beam (Figure 8(a)), which may be caused by the energy fluctuation of the UV beam, especially when the pulse energy is low.

We simulate the observed reduction of UV damage threshold based on the commonly used rate equation model with the inclusion of defect states:

$$
\begin{aligned}
& \frac{d n_{e}}{d t}=W_{M P I}(I)+\alpha I n_{e}+\sigma_{j} I^{j} n_{d}-\frac{n_{e}}{\tau_{e}} \\
& \frac{d n_{d}}{d t}=\frac{n_{e}}{\tau_{e}}-\sigma_{j} I^{j} n_{d}-\frac{n_{d}}{\tau_{d}}
\end{aligned}
$$

where $n_{e}$ is free electron density, $W_{\mathrm{MPI}}$ is the Keldysh multi-photon ionization rate, $I$ is laser intensity, $\alpha$ and $\sigma$ are the ionization cross section of avalanche electrons and defect states, respectively, $\tau_{e}$ and $\tau_{d}$ are decay time of free electrons and defect states, respectively, and $n_{d}$ is defect state density. We use $j=1$ and 2 for UV and IR beams respectively [15]. Both UV and IR pulses have a Gaussian shape in time and the pulse duration (FWHM) is $70 \mathrm{fs}$ and $60 \mathrm{fs}$, respectively. In our model, free electrons are first generated by photo-ionization (calculated by the Keldysh theory) and the electron density is further increased by avalanche ionization within the pulse duration. The decay time of these electrons is $150 \mathrm{fs}$ and all of them are assumed to decay into defect states which have a bandgap of $3.2 \mathrm{eV}$. It should be noted that in this model the defect states have a single band lying in between the conduction band and exciton band [15]. The second pulse re-ionizes the defect states to free electrons, and both photo and avalanche ionization are also considered. Damage occurs when free electron density reaches $1.7 \times 10^{21} \mathrm{~cm}^{-3}$ (critical density at $800 \mathrm{~nm}$ wavelength).

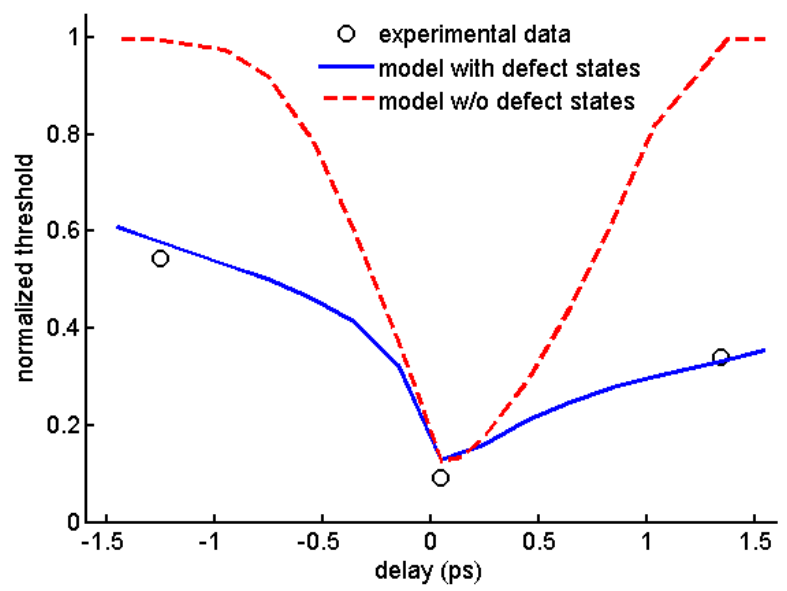

Fig. 9. Comparison of numerical calculation of damage threshold with and without defect states at different delays. Dots are experimental results from Fig. $8(37 \mu \mathrm{J}) . \alpha_{2}=20 \mathrm{~cm}^{2} / \mathrm{J}, \alpha_{6}=4 \mathrm{~cm}^{2} / \mathrm{J}, \sigma_{1}=2 \times 10^{-3}\left(\mathrm{~W} / \mathrm{m}^{2}\right) \mathrm{s}^{-1}, \sigma_{2}=1.1 \times 10^{-21}\left(\mathrm{~W} / \mathrm{m}^{2}\right)^{2} \mathrm{~s}^{-1}, \tau_{e}=150 \mathrm{fs}$, $\tau_{d}=1 \mathrm{ps}$, pulse duration (FWHM): 70 fs for UV and 60 fs for IR. 
The modeling results and experimental data (37 $\mu \mathrm{J}$, Fig. 8) are shown in Fig. 9, and for comparison, the modeling results using the same parameters without the defect states term is also shown. As can be seen, our model agrees with the experimental data well with the defect states term considered, and the lifetime of defect states is found to be $\sim 1 \mathrm{ps,}$ which is similar to recent results [20]. Without defect states, the damage threshold will return to its normal value at much shorter delays because the electrons have a shorter lifetime.

\subsection{Improving damage shape}

In this section we demonstrate that damage shape can be improved, and due to this improvement and the combination with the IR pulse, the required UV pulse energy can be reduced to sub-nJ level [21].

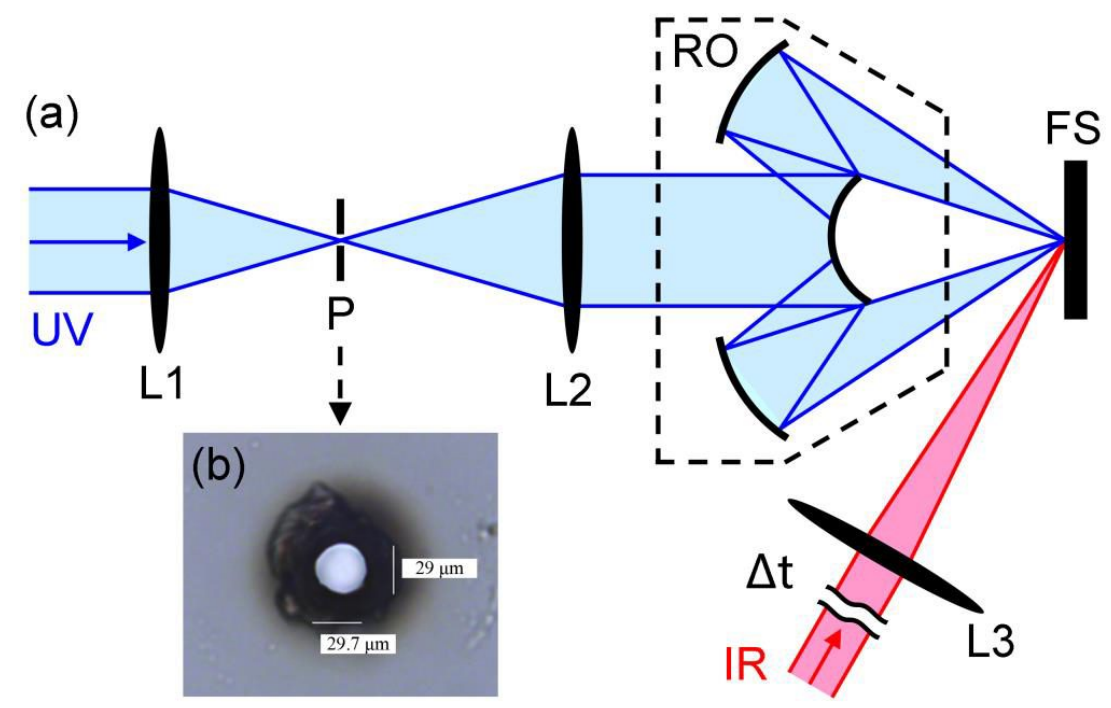

Fig. 10. (a) Experimental setup. L1-L3: plano-convex lenses. P: pinhole. RO: reflecting objective. FS: fused silica sample. $\Delta \mathrm{t}$ : mechanical delay line. (b) Optical image of a pinhole used in the experiments.

Figure 10 shows the experimental setup. The UV beam, generated from third harmonic generation (THG), first goes through a spatial filter which consists of two thin lenses ( $\mathrm{L} 1, \mathrm{f}=500 \mathrm{~mm}$ and $\mathrm{L} 2, \mathrm{f}=1 \mathrm{~m})$, and a pinhole drilled through a borosilicate microscope cover glass (thickness $150 \mu \mathrm{m}$ ) by another IR beam. The estimated UV pulse duration after L2 is $85 \mathrm{fs}$, due to dispersion of the two lenses. The diameter of the pinhole matches the calculated focal spot size of L1. Lenses L1 and L2 also form a $2 \times$ beam expander so that the filtered UV beam with a smooth Gaussian profile overfills the input aperture of the reflecting objective (RO, Edmund, 0.5 NA, working distance $23.2 \mathrm{~mm}$ ). The UV beam is then focused by the RO perpendicularly onto a fused silica sample (Corning 7980), with an estimated focal spot diameter of $0.64 \mu \mathrm{m}$. The sample is mounted on a motorized three-axis stage. The IR beam (60 fs FWHM) goes through a mechanical delay line and then is focused by L3 $(\mathrm{f}=200 \mathrm{~mm})$ to the sample at a $\sim 45^{\circ}$ incidence angle. The IR spot size (observed from a CCD camera) is $20 \times 15 \mu \mathrm{m}^{2}$ due to the oblique angle. The CCD camera monitoring the sample surface ensures that the UV spot remains at the center of the IR spot. The energy of the UV beam is controlled by a half-wave plate and a polarizer placed before the THG crystals, while the energy of the IR beam is controlled by a rotating neutral density (ND) filter. Both UV and IR energies are measured in front of the sample. The energy fluctuations of the UV and IR beams are within 5\% and 1\%, respectively. For temporal overlapping, multiple-shot UV damage threshold is measured at different delays (temporal step $60 \mathrm{fs}$ ) at a fixed IR pulse energy below its damage threshold and a slow sample moving speed of $0.4 \mathrm{~mm} / \mathrm{s}$, and the optimal delay ( $\sim 6 \mathrm{fs})$ is determined when the lowest UV damage threshold is observed. In the following single-shot measurements, the sample moves at a speed of $20 \mathrm{~mm} / \mathrm{s}$, ensuring that each UV-IR pulse pair irradiates at a fresh site. In all the cases no visual damage is seen with the IR beam alone. 


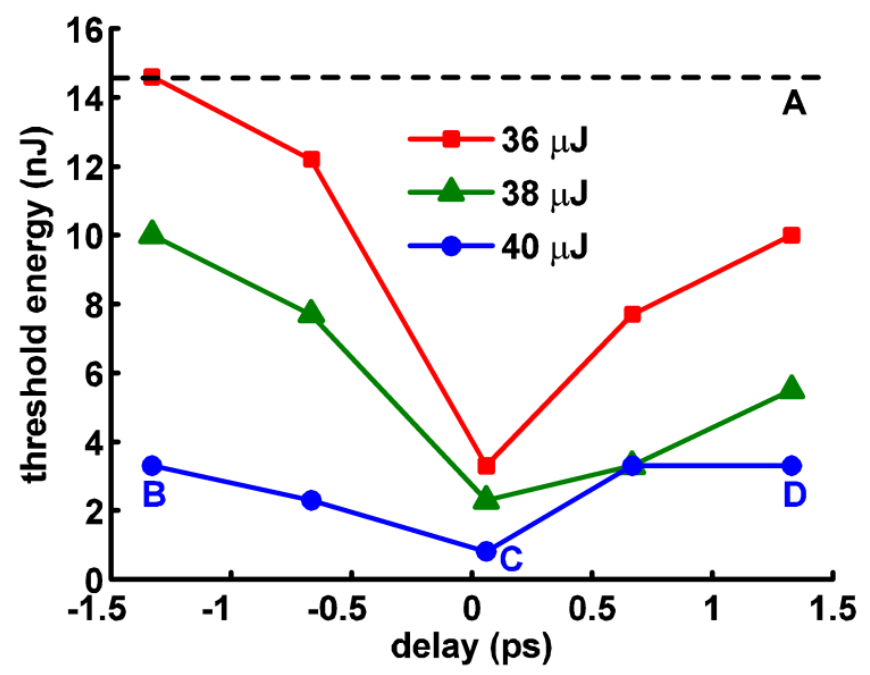

Fig. 11. UV damage threshold energy measured at different IR energies and delays. The black dashed line (A) indicates UV only threshold. SEM images corresponding to conditions A-D are shown in Fig. 12(a-d), respectively. Square, triangular and circular dots are experimental data. Solid lines are a guide to the eye.

At a certain delay and IR pulse energy, the single-shot UV damage threshold is determined as the lowest UV energy with which damage is observed using a scanning electron microscope (SEM). Twelve UV energies ranging from 0.4 to $19 \mathrm{~nJ}$ are used, and the results with five delays and three IR energies are shown in Fig. 11. The UV pulses arrive at the sample surface before and after the IR pulses at positive and negative delays, respectively. Theoretically, in ultrafast laser ablation of dielectrics, damage is formed when free electron density reaches a critical density. At positive delays, free electrons are first generated by the UV pulse through multiphoton ionization. These electrons either absorb energy from the following IR pulse at short delays ( $<300 \mathrm{fs})$ or first decay into self-trapped excitons and then are re-ionized by the IR pulse at long delays (>1 ps) [15]. The result in both cases is a rapid increase in free electron density due to avalanche ionization during the IR pulse duration, and eventually this density reaches the critical density, and damage is formed. With the help of the IR pulse, only a small number of free electrons (generated by the UV pulse) are needed to act as "seed electrons", and therefore the UV damage threshold is reduced. At negative delays, a similar process occurs except that the UV and IR pulses switch their roles.

The major difference of the results shown in Fig. 11 from those in Section 3.1 is reduced UV threshold energies, for both the UV only and the pulse train case. Specifically, the UV only threshold is reduced from $64 \mathrm{~nJ}$ to $14.6 \mathrm{~nJ}$, and the lowest UV threshold when combined with an IR pulse is reduced from $6 \mathrm{~nJ}$ to $0.8 \mathrm{~nJ}$. This reduction in threshold energy is attributed to the smaller focal spot and therefore smaller ablation area (shown below). Special attention is given to the case with $40 \mu \mathrm{J} \mathrm{IR}$ energy, with which the UV damage threshold is reduced to $0.8 \mathrm{~nJ}$, only $5.5 \%$ of the normal threshold value. To the best of our knowledge, it is the first time that such low energy is used in femtosecond ablation of dielectrics. It should be noted that, even with the improved UV beam, the damage threshold energy (14.6 nJ) is still 5 times higher than estimated $(2.8 \mathrm{~nJ})$ for a focal diameter of $0.64 \mu \mathrm{m}$ [22], perhaps due to a Bessel-like instead of a Gaussian profile of the UV focus (shown below in Fig. 12). Therefore, nano-ablation with even lower pulse energy is possible by replacing the reflecting objective with a transmitting objective, which generates an Airy pattern with more energy concentrated in the central spot compared to the Bessel-like pattern. It is worth noting that, although Fig. 11 emphasizes the reduction of UV damage threshold, these data can also be interpreted as reduction of IR threshold with the help of UV pulses. For example, Point B in Figure 4-2 indicates that, with $3.5 \mathrm{~nJ}$ UV pulse energy and -1.3 ps delay, the IR threshold is reduced from $42 \mu \mathrm{J}$ (IR only threshold) to $40 \mu \mathrm{J}$. 

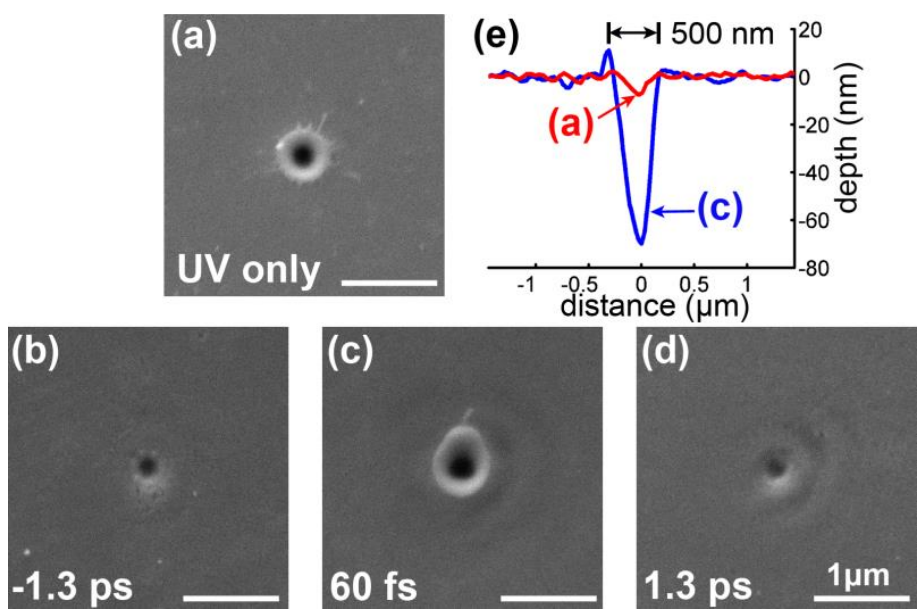

Fig. 12. (a-d) SEM images of the damage spots with UV beam only, UV-IR pulse train at $-1.3 \mathrm{ps}, 60 \mathrm{fs}$, and $1.3 \mathrm{ps}$ delay, respectively. (a-d) correspond to the conditions labeled A-D in Fig. 11, respectively. (e) Typical cross-sections of the damage in (a) and (c) measured with an atomic force microscope (AFM).

Figure 12 shows SEM images of the damage spots corresponding to A-D in Fig. 11, respectively, and typical crosssections for Fig. 12(a) and (c) are shown in Fig. 12(e). Craters with circular shapes are achieved with the aforementioned beam-shaping technique for both UV only [Fig. 12(a)] and the pulse train case [Fig. 12(b-d)]. An increase in crater depth is observed: from $7 \mathrm{~nm}$ with the UV beam alone [Fig. 12(a)] to $70 \mathrm{~nm}$ with the UV-IR pulse train at a 60 fs delay [Fig. 12(c)], while the damage size on the surface remains similar. Besides, Fig. 12(b) and (d) also show that craters fabricated with a pulse train tend to be smaller at longer delays. Although these two effects, namely the increase in ablation depth with pulse trains and the reduction in feature size at long delays, have been reported [13, 23], to the best of our knowledge, it is the first time to show these effects in nanomachining, and thus it indicates the possibility of controlling the size of fabricated nanostructures through pulse delays. The ripple structures adjacent to the central craters in Fig. 12(c) and (d) are attributed to the Bessel-like profile of the UV focus. Combining with the IR beam enhances ablation effects and therefore reveals the otherwise unseen structures with the UV beam only [Fig. 12(a)]. These structures can be removed when a transmitting objective lens is used as suggested earlier.

\section{CONCLUSION AND OUTLOOK}

In this paper, we use a pair of UV and IR pulses to generate damage on the surface of fused silica. With the energies of both pulses below their respective single-beam threshold, a 90-95\% reduction of UV damage threshold is achieved with the assistance of the IR pulse at $~$ zero delay. A rate equation model is used to help explain the experimental results. Two IR polarization states (linear and circular) are found to have similar effect on the UV damage threshold and resultant damage shape. Due to the higher damage threshold for circularly polarized IR beams, further reduction of UV damage threshold is achieved. The resultant damage by the combined action of UV and IR pulses retains the shape and size of the UV pulse, and with tightly focused UV pulses 500-nm diameter craters are fabricated with sub-nJ UV pulse energy. These results indicate the possibility of fabricating even smaller features with shorter wavelength pulses well below their damage threshold, and thus improving energy efficiency. In the current work, the reduction of UV damage threshold is achieved by combining a second pulse with near-infrared wavelength. Increasing the second pulse's wavelength (for example, to mid-infrared $(3-8 \mu \mathrm{m})$ [24]) might increase the avalanche effect, and thus further reduce UV damage threshold or reduce the required IR fluence.

\section{ACKNOWLEDGMENT}

This material is based on work supported by the Army Research Office. Partial support of this work from the National Science Foundation is also acknowledged. 


\section{REFERENCES}

[1] R.R. Gattass and E. Mazur, "Femtosecond laser micromachining in transparent materials," Nature Photon. 2, 219 (2008).

[2] M. Schultze, E.M. Bothschafter, A. Sommer, S. Holzner, W. Schweinberger, M. Fiess, M. Hofstetter, R. Kienberger, V. Apalkov, V.S. Yakovlev, M.I. Stockman, and F. Krausz, "Controlling dielectrics with the electric field of light," Nature 493, 75 (2013).

[3] P. Balling and J. Schou, "Femtosecond-laser ablation dynamics of dielectrics: basics and applications for thin films," Rep. Prog. Phys. 76, 36502 (2013).

[4] E.G. Gamaly, "The physics of ultra-short laser interaction with solids at non-relativistic intensities," Phys. Rep. 508, $91(2011)$.

[5] L. Sudrie, a. Couairon, M. Franco, B. Lamouroux, B. Prade, S. Tzortzakis, and a. Mysyrowicz, "Femtosecond laserinduced damage and filamentary propagation in fused silica," Phys. Rev. Lett. 89, 186601 (2002).

[6] J. Békési, J.H. Klein-Wiele, and P. Simon, "Efficient submicron processing of metals with femtosecond UV pulses," Appl. Phys. A 76, 355 (2003).

[7] D. Nikogosyan, M. Dubov, H. Schmitz, V. Mezentsev, I. Bennion, P. Bolger, and A. Zayats, "Point-by-point inscription of 250-nm-period structure in bulk fused silica by tightly-focused femtosecond UV pulses: experiment and numerical modeling," Cent. Eur. J. Phys. 8, 169 (2010).

[8] X. Yu, Q. Bian, B. Zhao, Z. Chang, P.B. Corkum, and S. Lei, "Near-infrared femtosecond laser machining initiated by ultraviolet multiphoton ionization," Appl. Phys. Lett. 102, 101111 (2013).

[9] Y. Wu, E. Cunningham, H. Zang, J. Li, M. Chini, X. Wang, Y. Wang, K. Zhao, and Z. Chang, "Generation of highflux attosecond extreme ultraviolet continuum with a 10 TW laser," Appl. Phys. Lett. 102, 201104 (2013).

[10] C.B. Schaffer, A. Brodeur, and E. Mazur, "Laser-induced breakdown and damage in bulk transparent materials induced by tightly focused femtosecond laser pulses," Meas. Sci. Technol. 12, 1784 (2001).

[11] K. Sugioka and K. Midorikawa, "VUV-UV multiwavelength excitation process for high-quality ablation of fused silica," Proc. SPIE 8777, 877704 (2013).

[12] M. Li, S. Menon, J.P. Nibarger, and G.N. Gibson, "Ultrafast electron dynamics in femtosecond optical breakdown of dielectrics," Phys. Rev. Lett. 82, 2394 (1999).

[13] I.H. Chowdhury, X. Xu, and A.M. Weiner, "Ultrafast two-color ablation of fused silica," Appl. Phys. A 83, 49 (2006).

[14] C.-H. Lin, Z.-H. Rao, L. Jiang, W.-J. Tsai, P.-H. Wu, C.-W. Chien, S.-J. Chen, and H.-L. Tsai, "Investigations of femtosecond-nanosecond dual-beam laser ablation of dielectrics," Opt. Lett. 35, 2490 (2010).

[15] D. Grojo, M. Gertsvolf, S. Lei, T. Barillot, D.M. Rayner, and P.B. Corkum, "Exciton-seeded multiphoton ionization in bulk $\mathrm{SiO}_{2}$," Phys. Rev. B 81, 212301 (2010).

[16] J.M. Liu, "Simple technique for measurements of pulsed Gaussian-beam spot sizes," Opt. Lett. 7, 196 (1982).

[17] D. Puerto, W. Gawelda, J. Siegel, J. Solis, and J. Bonse, "Erratum: "Plasma formation and structural modification below the visible ablation threshold in fused silica upon femtosecond laser irradiation" [Appl. Phys. Lett. 91, 082902 (2007)]," Appl. Phys. Lett. 92, 219901 (2008).

[18] Y.P. Raizer, Laser-Induced Discharge Phenomena, Consultant Bureau, New York, 1977.

[19] X. Yu, Q. Bian, Z. Chang, P.B. Corkum, and S. Lei, "Femtosecond laser nanomachining initiated by ultraviolet multiphoton ionization," Opt. Express 21, 24185 (2013).

[20] J. Peng, D. Grojo, D.M. Rayner, and P.B. Corkum, "Control of energy deposition in femtosecond laser dielectric interactions," Appl. Phys. Lett. 102, 161105 (2013).

[21] X. Yu, Z. Chang, P.B. Corkum, and S. Lei, "Fabricating nanostructures on fused silica using femtosecond infrared pulses combined with sub-nanojoule ultraviolet pulses," Opt. Lett. 39, 5638 (2014).

[22] T.Q. Jia, H.X. Chen, M. Huang, F.L. Zhao, X.X. Li, S.Z. Xu, H.Y. Sun, D.H. Feng, C.B. Li, X.F. Wang, R.X. Li, Z.Z. Xu, X.K. He, and H. Kuroda, "Ultraviolet-infrared femtosecond laser-induced damage in fused silica and $\mathrm{CaF}_{2}$ crystals," Phys. Rev. B 73, 054105 (2006).

[23] S. Zoppel, R. Merz, J. Zehetner, and G.A. Reider, "Enhancement of laser ablation yield by two color excitation," Appl. Phys. A 81, 847 (2005).

[24] D.M. Simanovskii, H.A. Schwettman, H. Lee, and A. J.Welch, "Midinfrared optical breakdown in transparent dielectrics," Phys. Rev. Lett. 91, 107601 (2003). 